\title{
The first human case of babesiosis mimicking Reiter's syndrome
}

\author{
Zuzana Strizova ${ }^{1}$, Klara Havlova ${ }^{2}$, Ondrej Patek ${ }^{3}$, Daniel Smrz ${ }^{1}$ and Jirina Bartunkova ${ }^{1}$ \\ ${ }^{1}$ Department of Immunology, Second Faculty of Medicine, Charles University and University Hospital Motol, Prague, Czech \\ Republic; \\ ${ }^{2}$ Department of Urology, Second Faculty of Medicine, Charles University and University Hospital Motol, Prague, Czech Republic; \\ ${ }^{3}$ Department of Internal Medicine, Second Faculty of Medicine, Charles University and University Hospital Motol, Prague, Czech \\ Republic
}

\begin{abstract}
Babesiosis is a tick-borne disease that may exhibit a broad range of clinical manifestations. According to the Food and Drug Administration (FDA), Babesia species belong to the most common transfusion-transmitted pathogens (FDA, May 2019), but the awareness of the disease caused by these parasitic protists is still low. In immunocompromised patients, the clinical course of babesiosis may be of extreme severity and may require hospital admission. We demonstrate a case of a young male who experienced severe polytrauma requiring repetitive blood transfusions. Six months later, the patient developed a classic triad of arthritis, conjunctivitis and non-specific urethritis. These symptoms largely mimicked Reiter's syndrome. The patient was later extensively examined by an immunologist, rheumatologist, urologist, and ophthalmologist with no additional medical findings. In the search for the cause of his symptoms, a wide laboratory testing for multiple human pathogens was performed and revealed a babesiosis infection. This was the first case of human babesiosis mimicking Reiter's syndrome. Following proper antimicrobial therapy, the patient fully recovered in four weeks. We aim to highlight that a search for Babesia species should be considered in patients with non-specific symptomatology and a history of blood transfusion or a possible tick exposure in pertinent endemic areas.
\end{abstract}

Keywords: arthritis, conjunctivitis, urethritis, Babesia microti, transfusion, diagnosis

Babesiosis is a tick-borne disease that may exhibit a broad range of clinical manifestations. The disease itself is a malaria-like zoonosis caused by protistan parasites of the genus Babesia Starcovici, 1893, which is transmitted usually after exposure to an infected tick (Ord and Lobo 2015). Another way of transmission is a transfusion of infected blood (Vannier and Krause 2012, Ord and Lobo 2015). The rate of transfusion-transmitted babesiosis (TTB) represents a growing concern (Villatoro and Karp 2019). Blood contaminated of Babesia species occurs in both endemic and non-endemic areas (Levin and Krause 2016). For that reason, a babesiosis-screening program for blood donations has been previously evaluated in the United States in the years 2010-2011. Despite the fact that PCR testing has shown significant efficacy in reducing the incidence of TTB, Babesia screening has not become a part of routine transmissible diseases-testing of donated blood in Europe (Young et al. 2012, Levin and Krause 2016). Moritz et al. (2014) revealed that screening for both Babesia-specific antibodies and DNA in blood donors might decrease TTB rates.
Different species of Babesia were reported to infect animals, but only B. microti (Franca, 1912) and B. divergens M'Fadyean et Stockman, 1911; B. venatorum Herwaldt, Cacciò, Gherlinzoni, Aspöck, Slemenda et al., 2003; B. duncani Conrad, Kjemtrup, Carreno, Thomford, Wainwright et al., 2006, and Babesia sp. MO1 strain (Herwaldt et al. 1996) can infect humans (Man et al. 2016). The geographic distribution of $B$. microti, B. duncani and $B$. venatorum is mostly associated with different areas of the United States. Babesia divergens was shown to reside in Europe, primarily in France and England. A study by Jiang et al. (2015) focused on the epidemiological characteristics of $B$. venatorum has demonstrated that forests followed by the courtyards and the public parks were the most common locations to receive a tick bite. Therefore, foresters were at a higher risk of becoming infected with $B$. venatorum. Moreover, these authors called for attention to be paid to the high rates of severe babesiosis manifestations in healthy non-splenectomised individuals (Jiang et al. 2015).

Address for correspondence: Zuzana Strizova, MD, Department of Immunology, Second Faculty of Medicine, Charles University and University Hospital Motol, V Úvalu 84, 15006 Praha 5, Czech Republic; phone +420 604712471; fax: 224435960; e-mail: zuzana.strizova@fnmotol.cz 
Table 1. Selected immunology and microbiology laboratory results of a young male infected with Babesia microti presenting with non-specific urethritis, conjunctivitis and arthritis.

\begin{tabular}{llc}
\hline & & reference ranges : \\
\hline IgG & $13.30 \mathrm{~g} / 1$ & $7.65-13.60$ \\
IgA & $4.02 \mathrm{~g} / 1$ & $0.91-2.90$ \\
IgM & $1.99 \mathrm{~g} / 1$ & $0.47-1.95$ \\
Specific IgE/Herbs & $0.74 \mathrm{kU} / 1$ & $0.00-0.35$ \\
ECP & $34.2 \mathrm{ng} / \mathrm{ml}$ & $0.0-24.0$ \\
ANA Ig 1:80 & negative & \\
ENA & negative & \\
ASCA & negative & \\
Rheumatoid factor & negative & \\
HLA B27 & negative & \\
HLA B5 & negative & \\
Eye smear and culture & Staphylococcus hominis \\
Oropharyngeal smear & Normal pharyngeal flora \\
Urethral smear & STD negative & \\
Chlamydia trachomatis & ELISA negative & \\
Chlamydia pneumoniae & ELISA negative & \\
Chlamydophila psitaci & Western blot negative \\
Yersinia enterocolica & ELISA negative \\
Mycoplasma pneumonie & IgG positive, IgM ELISA negative \\
Mannan serum detection & negative \\
Galactomannan detection & negative \\
Babesia microti & Elispot positive ** (8 SI) \\
\hline
\end{tabular}

Different species of Babesia were seen across the USA, Europe and Asia (Leiby 2011). In the USA, human babesiosis was initially reported in California, but later reported also in the area from coastal Rhode Island and New York to Upper Midwest, specifically Minnesota and Wisconsin, and other nearby geographical areas (Leiby 2011).

A study by Gabrielli et al. (2014) has shown a high prevalence of $B$. microti and $B$. divergens in professional groups, such as foresters, veterinary practitioners, hunters, and livestock keepers in Italy. The overall serological reactivity was higher in northern Italy than in central Italy, and livestock keepers represented the most seroreactive group (24\%, mainly reactive to B. divergens and B. bovis (Babes, 1892) (Gabrielli et al. 2014). In that study, no cross-reactivity was observed among different babesial antigens.

In the Czech Republic, the first case of human babesiosis was reported in 2003. To our knowledge, it has also been the first case of symptomatic infection with $B$. microti imported to Europe from the United States (Nohýnková et al. 2003). Babesiosis may be clinically presented in diverse forms depending on the immune competency of the patient. In young healthy individuals, babesiosis is usually asymptomatic or exhibits just a few non-specific flu-like symptoms, such as fever, fatigue or myalgia (Homer et al. 2000). Among less common symptoms were also described arthralgia, conjunctival injection, and hyperesthesia (Vannier et al. 2015). It has also been noted that hepatomegaly occurs upon Babesia infection (Vannier et al. 2015).

In the immunocompromised patients, the clinical course of babesiosis may be of extreme severity and may require hospital admission (Vannier and Krause 2012, Krause 2019). It has been demonstrated that in patients who had undergone splenectomy, the infection with $B$. microti may lead to severe heart, lung and liver disorders (Vannier and Krause 2012). In cases where infection with species of Babesia is suspected, obtaining the diagnosis can be achieved by microscopic evaluation of blood smear, PCR or immunofluorescent assays (Vannier and Krause 2012).

Here we present a rare case of a 36-year old male who was examined at the Department of Immunology at the University Hospital Motol, Prague for recurrent urologic and ophthalmologic symptoms. The main complaints included dysuria and periurethral itch and mild oedema of the eye lids, also accompanied by an itch. The patients' medical history did not reveal any atypical events. However, the patient experienced a motorcycle accident six months before immunology examination and was hospitalised with a polytrauma for four weeks. During hospitalisation, the patient obtained multiple blood transfusions. After he fully recovered, his urologic symptoms appeared. The patient did not visit a medical facility for five months because the symptoms were mild, and he believed they were associated with the previously inserted permanent urinary catheter. Nevertheless, the intensity of the symptoms rapidly increased and the ophthalmologic symptoms appeared.

We have examined the patient, who did acknowledge a mild pain in the right hypochondrium. Furthermore, the patient presented with reddish eyelids and conjunctives. The rest of the examination did not show any signs of pathology. The patient's blood was taken in order to run the immunology tests. The extended set of immunological testing included: detection of serum levels of antibodies (isotypes $\operatorname{IgG}, \operatorname{IgA}, \operatorname{IgM}$, and $\operatorname{IgE}$ ); the identification of autoantibodies - Antinuclear Antibodies (ANA), Extractable Nuclear Antigens Antibodies (ENAs), Anti-Saccharomyces cerevisiae Antibodies (ASCA) and multiple other autoantibodies that are frequently associated with autoimmune diseases (RF, ASMA, GPCA, LKM, AECA, GBM, ANCA, etc.) (Suurmond and Diamond 2015), and the measurement of basic cellular immunity parameters $\left(\mathrm{CD}^{+}\right.$ cell count, $\mathrm{CD}^{+}{ }^{+} \mathrm{CD} 4^{+}$cell count, $\mathrm{CD} 3^{+} \mathrm{CD}^{+}$cell count, $\mathrm{CD}^{-}{ }^{-\mathrm{CD}} 16^{+} \mathrm{CD}^{2} 6^{+}$cell count, $\mathrm{CD} 19^{+}$cell count, $\mathrm{CD}^{+} /$ $\mathrm{CD}^{+}$cell ratio). The patient was also advised to visit a venereologist and an ophthalmologist for a detailed analysis. Therefore, urethra smear evaluation and cell culture were performed, and also the conjunctival and throat swab scheduled.

Two months later, the patient came for a control checkup and the lab results (Table 1). There was no detected pathology in the immunology results, nor in the venerology/ophthalmology results, which referred to non-specific urethritis and non-specific conjunctivitis. The immunology tests showed only mild elevation of specific IgE antibodies against herbs and elevated eosinophilic cationic protein. The patient began to use antihistamine medications. Nevertheless, in a few days, a new symptom appeared and led us to the possible diagnosis of Reiter's syndrome.

The patient newly suffered from joint pain and, therefore, was thought to complete the triad - arthritis, conjunctivitis and urethritis, known as Reiter's syndrome. The rheumatologic examination was scheduled. The pelvis X-Ray, sacroiliacal joints X-ray and knee joints X-ray were made. However, together with the genetically confirmed HLA B27 and HLA B5 negativity, the tests did not support 


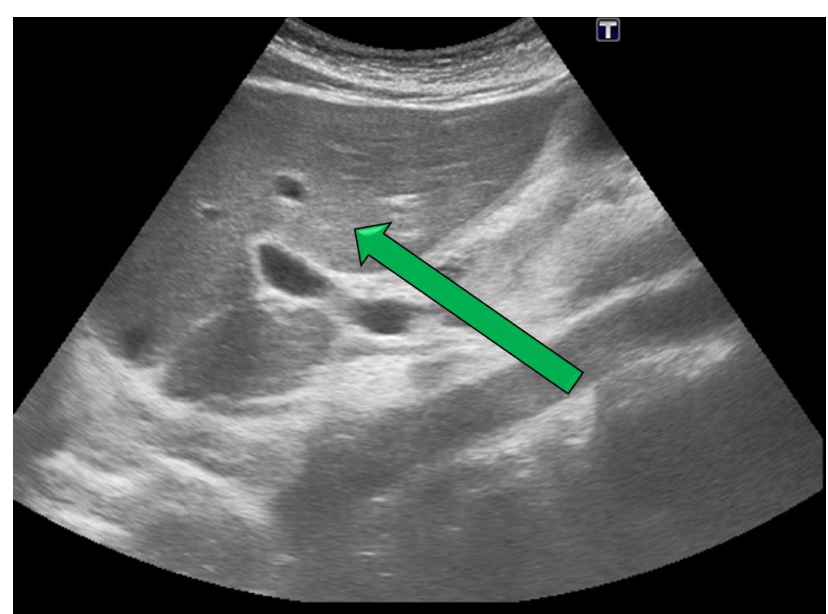

Fig. 1. The abdominal ultrasonography showed slightly enlarged liver (hepatomegaly) with adequate echogenicity and without focal changes in the parenchyma (green arrow). Abdominal cavity did not display the presence of free fluid. Convincing pathological expansions or enlarged lymph nodes were not seen by ultrasonography imaging.

the diagnosis of Reiter's syndrome. The patient was admitted to the Department of Internal Medicine and extensively examined in the search for infectious agents. Also, a wide spectrum of biochemical testing was performed. The chest X-ray did not reveal pathology. However, the abdominal ultrasound showed hepatosplenomegaly (Figs. 1, 2). Dysuria and eyelid swelling became clinically unbearable for the patient.

Based on the clinical appearance, the patient was initially advised to undergo laboratory testing, such as enzyme-linked immunosorbent assay (ELISA) and Western Blot, for multiple pathogens, including Chlamydia trachomatis, Chlamydophila pneumoniae, Mycoplasma pneumoniae, Chlamydophila psittaci and Yersinia enterocolitica. Polymerase chain reaction (PCR)-based methods were further used for the detection of Candida (mannan antigen) and Aspergillus (galactomannan antigen). All tests, however, were negative. Borrelia serology was not provided at our institution due to the exclusion of possible tick exposure. The patient was confident about not having any contact with infected ticks and claimed he certainly had not been exposed to the wild nature, nor ticks. Due to a high prevalence of Borrelia antibodies in healthy individuals, the serological testing against Borrelia could have been misleading (Strizova et al. 2020).

Various methods have been employed for the identification of Babesia spp. Both microscopic (blood smear evaluation) and serological methods represent a useful tool for routine diagnosis of babesiosis (Parija et al. 2015). Culture techniques appear to provide highly variable results and remain time-consuming (Parija et al. 2015). In our patient, the babesiosis infection was confirmed by Lymphocyte Transformation Test (LTT, ELISPOT) (Table 1). The LTT is a highly sensitive immunoassay that allows a rapid analysis of produced antibodies (biotinylated and followed by a streptavidin-enzyme conjugate) and secreted-cytokines by immune cells in the presence or absence of stimuli ( $\mathrm{Ji}$ et

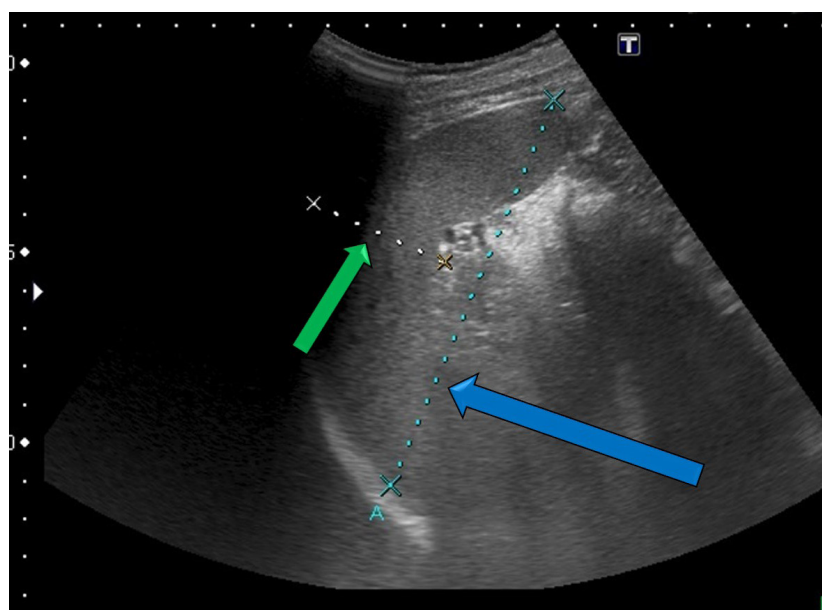

Fig. 2. Mild enlargement of the spleen (splenomegaly) was verified by ultrasonography imaging, as shown in longitudinal (blue arrow) and transversal (green arrow) diameters. Both diffuse hepatomegaly and splenomegaly were previously reported in babesiosis.

al. 2016). The LTT technology, therefore, represents one of the most effective diagnostic approaches to detect and quantify low-frequency cytokine- or antibody-producing cells and has been approved by the Food and Drug Administration (FDA) for use in the diagnosis of tuberculosis (Jin et al. 2013, Ji et al. 2016).

In Lyme disease, the sensitivity of the LTT in clinical borreliosis was determined as $89.4 \%$, and the specificity was $98.7 \%$ (von Baehr et al. 2012). Moreover, LTT has been shown to provide superior sensitivity and specificity as compared to Western blot. Whereas Western blot has been reported to provide frequently false-positive results, including cross-reactivity with other infectious diseases, LTT did not reveal cross-reactivity with other pathogens (Jin et al. 2013). In babesiosis, sensitivity and specificity of LTT in the diagnosis have not yet been evaluated, so further studies on the relevance of Babesia LTT are needed.

With respect to the concluded diagnosis of human babesiosis, the patient was advised to undergo antimicrobial treatment. The routine antimicrobial treatment for human babesiosis includes atovaquone and azithromycin, or a combination of quinine and clindamycin (Vannier and Krause 2012, Vannier et al. 2015). However, in chronic babesiosis, trimethoprim-sulfamethoxazole was found to be also effective (Vannier et al. 2015). The patients' therapy, therefore, consisted of doxycycline $(200 \mathrm{mg} /$ per day) and trimethoprim-sulfamethoxazole (160/800/per day), which is recommended in immunosuppressed patients suffering babesiosis (Vannier et al. 2015). The length of the therapy was 28 days. Although the patient obtained his final diagnosis and treatment at different institutions, the control check-up was arranged at the University Hospital Motol in Prague. By that time, the patient did not display any clinical symptoms. This complete remission was observed four weeks after the initiation of the therapy. The patient did not schedule any other hospital visits, and due 
to the lack of symptomatology did not require further examinations.

Babesiosis is a tick-borne disease that can be clinically presented in diverse forms. According to the FDA, Babesia spp. represent a group of pathogens that are frequently transmitted by blood transfusion (Levin and Krause 2016). Human babesiosis is, however, among the most challenging parasitic infections to diagnose. The clinical appearance can range from mild flu-like symptoms to severe and life-threatening organ disorders. This represents a significant hurdle since the common awareness of the disease is still low (Krause et al. 2003, Kwon et al. 2018).

In this report, we presented a case of a young male who experienced severe polytrauma requiring repetitive blood transfusions. Six months later, the patient developed a classic triad of arthritis, conjunctivitis, and non-specific urethritis. These symptoms largely mimicked Reiter's syndrome. The patient was later extensively examined by an immunologist, rheumatologist, urologist, and ophthalmologist with no additional medical findings. The patient's condition still deteriorated and finally required hospitalisation at the Department of Internal Medicine. The patient's subjective complaints, such as arthralgia and myalgia, together with objective inflammation of the urethra, non-specific conjunctivitis, and ultrasound-verified hepatosplenomegaly, were the only medical findings during his hospitalization. In the search for the cause of his symptoms, a wide laboratory testing for multiple human path- ogens was performed and revealed a babesiosis infection. Following proper antimicrobial therapy, the patient fully recovered in four weeks.

Symptoms such as arthralgia and conjunctival injection have already been described in patients with babesiosis. However, in this report, we demonstrate the first case of human babesiosis mimicking Reiter's syndrome with the classic triad of arthritis, conjunctivitis and urethritis.

Although it has already been proven that PCR screening is effective in reducing the incidence of TTB, there is no specific Babesia screening program for blood donations in the Czech Republic. Moreover, these screening programs are not routinely applied in European countries. Most of Babesia-infected immunocompetent patients experience mild symptoms and do not require examination or further treatment. However, we would like to stress that patients in need of blood transfusion may often have an alteration of the immune functions and, therefore, might be at significantly higher risk of developing severe babesiosis. Since there is no screening program preventing the TTB, a specific search for Babesia species should be conducted in patients with non-specific symptomatology and a history of blood transfusion or a possible tick exposure in pertinent endemic areas.

Acknowledgements. We would like to thank the doctors, nurses, and other medical staff for their professional assistance throughout the study.

\section{REFERENCES}

von Baehr V., Doebis C., Volk H.D., Von Baehr R. 2012: The lymphocyte transformation test for Borrelia detects active Lyme borreliosis and verifies effective antibiotic treatment. Open Neurol. J. 6: 104-112.

Gabrielli S., Calderini P., Cassini R., Galuppi R., Tampieri M.P., Pietrobelli M., Cancrini G. 2014: Human exposure to piroplasms in Central and Northern Italy. Vet. Ital. 50: 41-47.

Herwaldt B., Persing D.H., Precigout E.A, Goff W.L., Mathiesen D.A., Taylor P.W., Eberhard M.L., GorenFLOT A.F. 1996: A fatal case of babesiosis in Missouri: identification of another piroplasm that infects humans. Ann. Intern. Med. 124: 643-650.

Homer M.J., Aguilar-Delfin I., Telford S.R. III., Krause P.J., Persing D.H. 2000: Babesiosis. Clin. Microbiol. Rev. 13: 451-469.

Ji N., Forsthuber T.G. 2016: ELISPOT Techniques. Methods Mol. Biol. 1304: 63-71.

Jiang J.F., Zheng Y.C., Jiang R.R., Li H., Huo Q. B., Jiang B.G., Sun Y., Jia N., Wang Y.W., Ma L., LiU H.B., Chu Y.L., Ni X.B., Liu K., Song Y.D., Yao N.N., Wang H., Sun T., CAO W.C. 2015: Epidemiological, clinical, and laboratory characteristics of 48 cases of "Babesia venatorum" infection in China: a descriptive study. Lancet Infect. Dis. 15: 196-203.

Jin C., Roen D.R., Lehmann P.V., Kellermann G.H. 2013: An enhanced ELISPOT assay for sensitive detection of Antigen-specific T Cell responses to Borrelia burgdorferi. Cells 2: 607-620.

Krause P.J. 2019: Human babesiosis. Int. J. Parasitol. 49: 165-174. Krause P.J., McKay K., Gadbaw J., Christianson D., Closter L., Lepore T., Telford S.R., Sikand V., Ryan R., Persing D., RADOlF J.D., Spielman A. 2003: Increasing health burden of human babesiosis in endemic sites. Am. J. Trop. Med. Hyg, 63: 431-436.

Kwon H.Y., Im J.H., Park Y.K., Durey A., Lee J.S., Baek J.H. 2018: Two imported cases of babesiosis with complication or co-infection with Lyme disease in Republic of Korea. Kor. J. Parasitol, 56: 609-613.

Leiby D.A. 2011: Transfusion-transmitted Babesia spp.: bull's-eye on Babesia microti. Clin. Microbiol. Rev. 24: 14-28.

Levin A.E., Krause P.J. 2016: Transfusion-transmitted babesiosis: is it time to screen the blood supply? Curr. Opin. Hematol. 23: 573-580.

Man S.Q., Qiao K., Cui J., Feng M., Fu Y.F., Cheng X.J. 2016: A case of human infection with a novel Babesia species in China. Infect. Dis. Poverty 5: 28.

Moritz E.D., Winton C.S., Johnson S.T., Krysztof D.E., Townsend R.L., Foster G.A., Devine P., Molloy P., BrisSette E., Berardi V.P., Stramer S.L. 2014: Investigational screening for Babesia microti in a large repository of blood donor samples from nonendemic and endemic areas of the United States. Transfusion 54: 2226-2236.

NohÝnková E., KubeK J., MěšŤánková O., Chalupa P., HubÁleK Z. 2003: [A case of an infection with Babesia microti imported to the Czech Republic from the USA]. Časopis lékařu českých 142: 377-381. (In Czech.)

ORD R.L., Loвo C.A. 2015: Human babesiosis: pathogens, prevalence, diagnosis and treatment. Curr. Clin. Microbiol. Rep. 2: $173-181$.

Parija S.C., Kp D., Venugopal H. 2015: Diagnosis and management of human babesiosis. Trop. Parasitol. 5: 88-93.

Strizova Z., Smrz D., Bartunkova J. 2020: Seroprevalence of Borrelia IgM and IgG antibodies in healthy individuals: a cau- 
tion against serology misinterpretations and unnecessary antibiotic treatments. Vect. Borne Zoon. 20: 10.

Suurmond J., Diamond B. 2015: Autoantibodies in systemic autoimmune diseases: specificity and pathogenicity. J. Clin. Invest. 125: 2194-2202.

Vannier E., Krause P.J. 2012: Human babesiosis. N. Engl. J. Med. 366: 2397-2407.

Vannier E.G., Diuk-Vasser M.A., Ben Mamoun C., Krause P.J. 2015: Babesiosis. Infect. Dis. Clin. North Am. 29: 357-370.
Villatoro T., KarP J.K. 2019: Transfusion-transmitted babesiosis. Arch. Pathol. Lab. Med. 143: 130-134.

Young C., Chawla A., Berardi V., Padbury J., Skowron G., Krause P.J. 2012: Babesia testing investigational containment study group. Preventing transfusion-transmitted babesiosis: preliminary experience of the first laboratory-based blood donor screening program. Transfusion 52: 1523-1529.

Cite this article as: Strizova Z., Havlova K., Patek O., Smrz D., Bartunkova J. 2020: The first human case of babesiosis mimicking Reiter's syndrome. Folia Parasitol. 67: 031. 